\title{
Real-time GPS Ionospheric TEC Estimation over South Korea
}

\author{
Byung-Kyu Choi ${ }^{1 \dagger}$, Sung-Moon Yoo ${ }^{1}$, Kyoung-Min Roh ${ }^{1}$, Sang-Jeong Lee ${ }^{2}$ \\ ${ }^{1}$ Korea Astronomy \& Space Science Institute, Daejeon 305-348, Korea \\ ${ }^{2}$ Chung-Nam National University, Daejeon 305-764, Korea
}

Ionosphere is one of the largest error sources when the navigational signals produced by Global Positioning System (GPS) satellites are transmitted. Therefore it is very important to estimate total electron contents (TEC) in ionosphere precisely for navigation, precise positioning and some other applications. When we provide ionospheric TEC values in real-time, its application can be expanded to other areas. In this study we have used data obtained from nine Global Navigation Satellite System (GNSS) reference stations which have been operated by Korea Astronomy and Space Science Institute (KASI) to detect ionospheric TEC over South Korea in real-time. We performed data processing that covers converting $1 \mathrm{~Hz}$ raw data delivered from GNSS reference stations to Receiver INdependent Exchange (RINEX) format files at intervals of 5 minutes. We also analyzed the elevation angles of GPS satellites, vertical TEC (VTEC) values and their changes.

Keywords: GPS, ionosphere, TEC, real-time

\section{INTRODUCTION}

The ionosphere influences every user that uses navigation signals, and is the largest error in signal transmission processes. When signals from navigation satellites pass the ionosphere, the navigation signals experience refraction, reflection, and attenuation by the total electron content (TEC) of the ionosphere.

A global positioning system (GPS) reference station network, which is distributed globally or locally, plays an important role in the precise calculation of ionospheric TEC (Skone 1998, Fedrizzi et al. 2005). Especially, with the development of information and communication technology and the Internet, GPS data can be received in real time from a remote location. The data obtained in real time is widely used for real-time ionosphere studies as well as highprecision positioning (Mannucci et al. 1998, Gao \& Liu 2002).

Studies on the analysis of the ionospheric characteristics are essential for precise positioning of users. In general, single-frequency GPS users utilize the Klobuchar model to estimate ionospheric delay error (Klobuchar 1987). On average, this model can correct ionospheric delay error by up to about $50 \%$ (Komjathy 1997). As the error from the ionosphere cannot be completely removed, single-frequency GPS users are not capable of precise positioning. In addition, the ionospheric TEC disturbance caused by increased solar activity and geomagnetic storms has large effects on navigation signals such as signal interruption or the increase in error. Ionosphere studies using GPS are also used to analyze the characteristics of earthquakes (Heki 2011, Artru et al. 2005). In the case of the 2010 Chile Earthquake (February 2010) and the Great East Japan Earthquake (March 2011), enormous energy was generated from the earthquake epicenter, and was transmitted to the atmosphere. Waves transmitted to the upper atmosphere in various forms affect ionospheric TEC, and thus the characteristics of the wave forms generated by earthquakes (e.g., Rayleigh surface wave and tsunami wave) could be analyzed. Therefore, the intensity or characteristics of earthquakes could be examined through the detection of the variation of TEC.

Recently, studies have been conducted to detect the variation of the ionosphere quickly and minutely (Spogli et al. 2009, Prikryl et al. 2013). Especially, Prikryl et al. (2013) used $1 \mathrm{~Hz}$ and $50 \mathrm{~Hz}$ GPS data to detect the variation of (c) This is an open Access article distributed under the terms of the Creative Commons Attribution Non-Commercial License (http:// creativecommons.org/licenses/by-nc/3.0/) which premits unrestricted non-commercial use, distribution, and reproduction in any medium, provided the original work is properly cited.
Received Jun 28, 2013 Revised Aug 5, 2013 Accepted Aug 9, 2013 †Corresponding Author

E-mail: bkchoi@kasi.re.kr

Tel: +82-42-865-3237, Fax: +82-42-861-5610 
TEC caused by geomagnetic storms at high latitudes. They observed scintillation, and also performed verification research using the radar and ionosonde observation data.

Blewitt et al. (2009) emphasized the use of $1 \mathrm{~Hz}$ GPS data for real-time crustal deformation monitoring and tsunami warning, which are caused by earthquakes. Also, Komjathy et al. (2012) showed that it is possible to monitor the variation of ionospheric TEC caused by earthquakes using a realtime GPS observation network, which is distributed globally. This indicates that the future research on and demand for ionospheric TEC are focused on real-time monitoring.

In this study, the strategies for the real-time monitoring of ionospheric vertical TEC (VTEC) are introduced, and the calculation results of ionospheric VTEC values during 1 hour are presented, which use the $1 \mathrm{~Hz}$ data from the nine global navigation satellite system (GNSS) reference stations operated by the Korea Astronomy and Space Science Institute. In addition, the time series of the variation of VTEC values is also provided.

\section{DATA PROCESSING METHODS}

To estimate ionospheric TEC precisely, the use of dual frequency GPS observation data (L1 1575.42 MHz and L2 1227.60 MHz) is essential. The receivers of GNSS reference stations, which are fixed on the ground, receive and store the GPS observation data that consists of code and carrier phase. The methods for calculating TEC using GPS observation data have been introduced in detail in a number of papers (Calais \& Minster 1998, Mannucci et al. 1999, Davis \& Hartmann 1997, Afraimovich et al. 2001, Otsuka et al. 2002). When GPS navigation signals pass through the ionosphere, signal delay occurs due to the free electrons in the ionosphere. This delay can be easily calculated using Eq. (1) (Sardon et al. 1994).

$$
S T E C=\frac{1}{40.308}\left[\frac{F_{1}^{2} \times F_{2}^{2}}{F_{1}^{2}-F_{2}^{2}}\right]\left(P_{2}-P_{1}+B^{s}+B_{r}\right)
$$

where $P_{i}(i=1,2)$ is the observed pseudorange value, and $F_{i}(i=1,2)$ is the GPS signal frequency. $B^{s}$ and $B_{r}$ represent the differential code bias (DCB) of the satellite and the receiver DCB, respectively. STEC represents the ionospheric TEC in the line-of-sight direction, and this value is converted to VTEC using an appropriate mapping function. Also, in this study, a carrier phase smoothing method, which uses both code and carrier phase, was utilized to increase the accuracy of TEC (Kleusberg 1986).

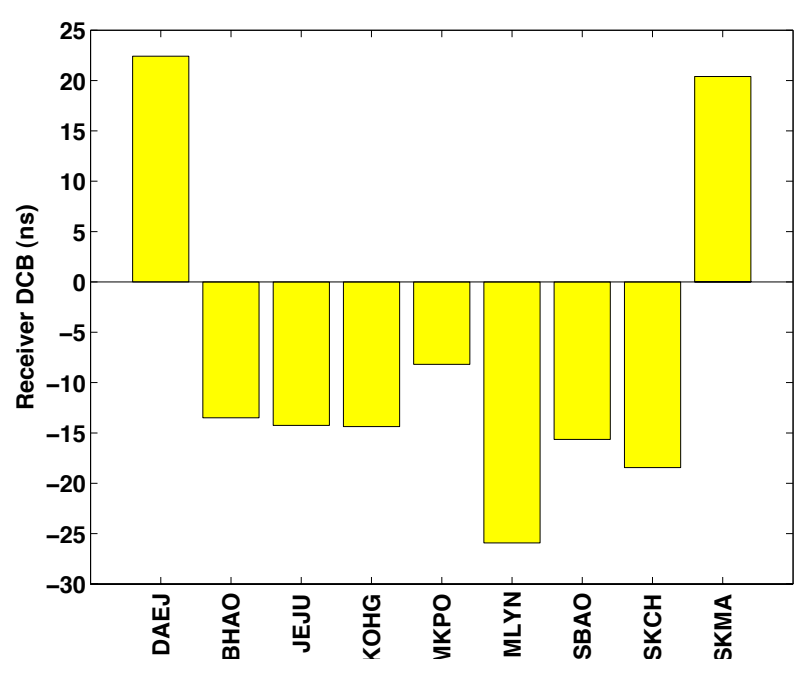

Fig. 1. Receiver DCB values (C1-P2) applied for the real-time ionospheric TEC estimation

DCB is the largest error in calculating ionospheric TEC with the use of observed dual frequency GPS values. Moreover, several GPS receivers have a DCB value of dozens of nanoseconds (ns), and this should be calculated for an accurate TEC calculation (Choi et al. 2011). If the DCB value is not accurately compensated, the reliability of the estimated TEC value would decrease. In this study, the DCB values calculated in advance (i.e., the day before) were applied to the real-time TEC calculation. Fig. 1 shows the estimation of the receiver DCB values for the nine GNSS reference stations operated by the Korea Astronomy and Space Science Institute, which were estimated in advance for the TEC calculation. The Daejeon (DAEJ) and Seoul (SKMA) GNSS reference station receivers (Trimble NetRS) had a DCB value of more than $20 \mathrm{~ns}$, and the other GNSS reference station receivers (Trimble NetR9) had a minus (-) value. Especially, the Milyang (MLYN) reference station receiver had a large DCB value (about $-26 \mathrm{~ns}$ ), which could have a large effect on the TEC calculation.

Fig. 2 shows the flow chart of the data processing for the real-time TEC calculation. The nine GNSS reference stations operated by the Korea Astronomy and Space Science Institute are evenly distributed over the Korean peninsula. Some of them are connected with the data center in Daejeon via the Internet network, and the others are connected with the data center in Daejeon via the dedicated lines. The GNSS reference stations receive the GPS signals in real time, and transmit the raw data (binary type) to the data center. The raw data followed the Radio Technical Commission for Maritime Service (RTCM) 3.0 data transfer standard protocol, and the Networked Transport of RTCM via Internet Protocol (NTRIP) 


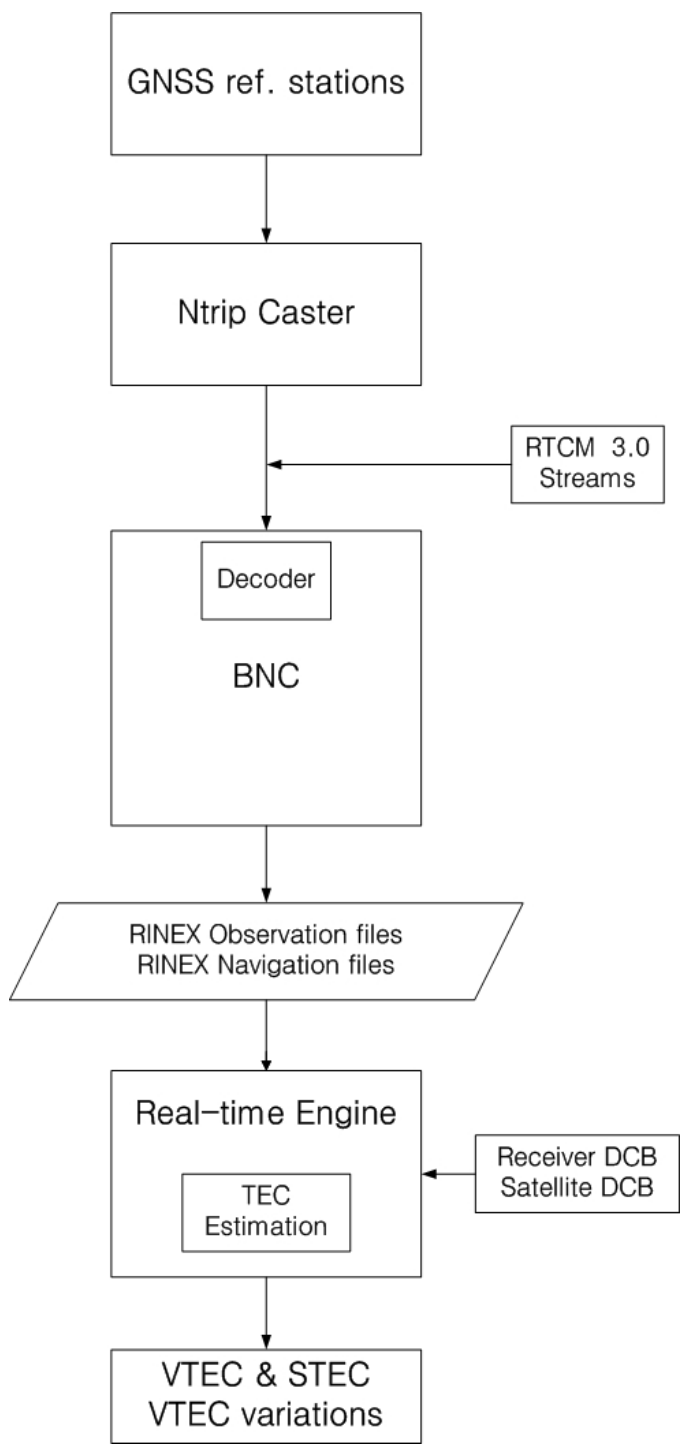

Fig. 2. A process flow diagram for the real-time ionospheric TEC calculation.

caster was used to control the transfer and flow of the GNSS data on the Internet.

As the RTCM data transmitted from NTRIP is raw data and is encoded, the decoding and conversion of this information are required. In this study, the BKG Ntrip Client (BNC) software version 2.7 developed by the Federal Agency for Cartography and Geodesy (BKG) in Germany was used. The BNC software examines the quality of GNSS data, and performs the conversion of raw data to Receiver Independent EXchange Format (RINEX) so that users can easily understand. Thus, the most basic form of data for the TEC calculation can be obtained using the BNC software. If the BNC software is not available, users can calculate TEC by directly converting the RTCM information that is transmitted

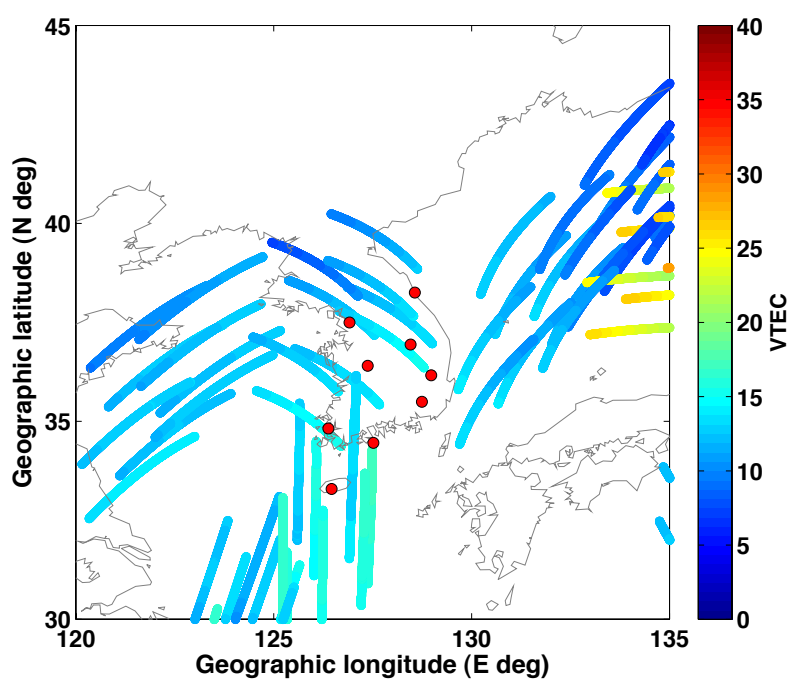

Fig. 3. Ionospheric pierce points for one hour and TEC variations.

to NTRIP. In this case, the RTCM information received at each port needs to be checked continuously, and the data needs to be converted and stored in a separate buffer.

The RINEX file was generated at an interval of 300 seconds, and the data was stored every second. In other words, the TEC values were calculated every 300 seconds, and TEC was calculated at each observation time. If the data from nine GNSS reference stations is used and every reference station receives 10 GPS satellite signals, the total number of TECs, which are to be calculated every 5 minutes, is $27,000=(=9 \times 10 \times 300)$. The information that is acquired at the end includes the TEC values in the vertical direction and the line-of-sight direction, and the variation of TEC depending on time. Especially, a real-time ionospheric TEC monitoring study focuses on the variation of TEC values rather than the TEC values themselves.

\section{REAL-TIME IONOSPHERIC TEC ESTIMATION}

To obtain ionospheric TEC values over the Korean peninsula and monitor the variation, the GPS observation data received at the GNSS reference stations on January 1, 2013 was used. The raw data from the nine GNSS reference stations was transmitted to the data center every second, and the RINEX file, which includes the $1 \mathrm{~Hz}$ observation data, was generated at an interval of 5 minutes at the data center. Using this GPS observation data from the nine GNSS reference stations that was stored every 5 minutes, the TEC values over the Korean peninsula during 1 hour were obtained as shown in Fig. 3. The data processing period was from 0:00 UT 
(09:00 KST) to 1:00 UT (10:00 KST). Fig. 3 shows the obtained variation of the VTEC values depending on the ionospheric pierce point (IPP) of the GPS navigation signals. Also, the small dots represent the position of the GNSS reference stations.

To minutely analyze the VTEC values estimated using the GPS observation data, the change in the elevation angle of satellites, the VTEC values, and the variation of the VTEC values depending on time were obtained for each GPS satellite. Figs. $4 \mathrm{a}-4 \mathrm{~d}$ show the data processing results of the GPS satellite pseudo random noise (PRN) 4, 20, 23, and 32 , respectively. For the satellite PRN 4, the elevation angle increased by about 18 degrees from 32 degrees to 50 degrees, and the VTEC value increased by 1 TEC unit (TECU) in the

PRN 4
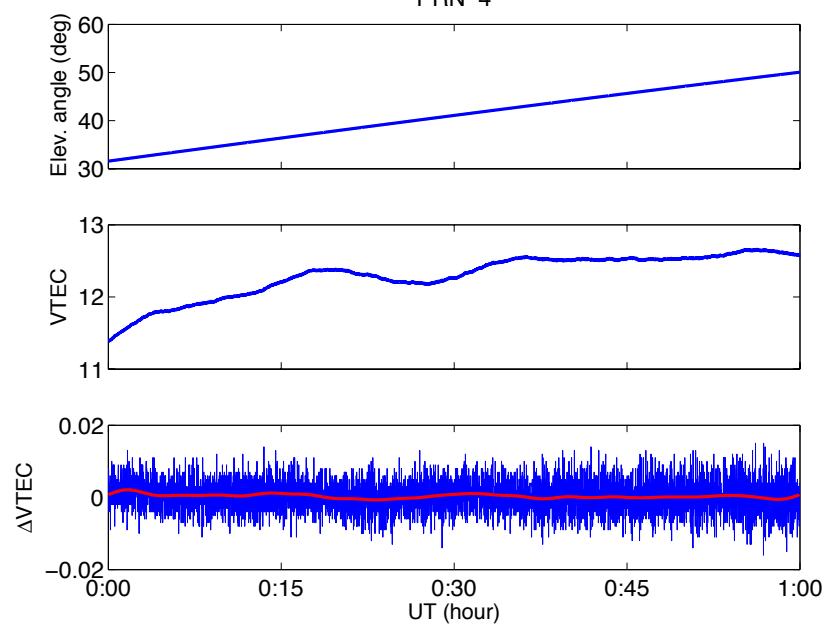

(a)
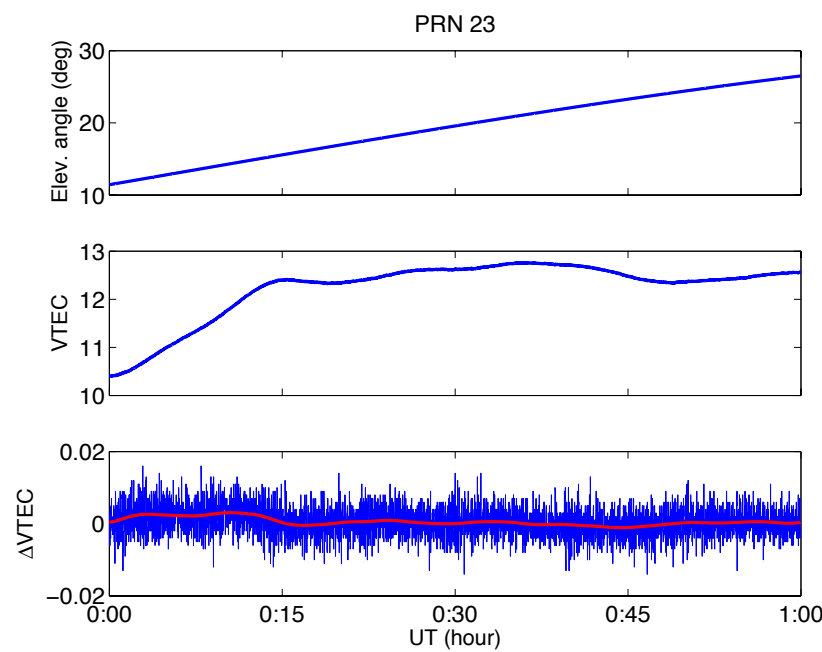

(c) first 20 minutes ( 1 TECU is $10^{16}$ electrons $/ \mathrm{m}^{2}$, which indicates that there are $10^{16}$ free electrons per square meter). Then the value remained stable during the next 40 minutes without a large variation. Also, the variation of the VTEC values (i.e., $\left.\left(V T E C_{n+1}-V T E C_{n}\right) / 1 \mathrm{sec}\right)$ was \pm 0.01 TECU. In other words, the time variation of the VTEC values was insignificant, and $\sum K_{p}$, the geomagnetic index for this day, was $0+$, which indicates that the geomagnetic activity itself was very stable. The Dst index was also found to be very stable using the data from the website of the world geomagnetic data center (http:// wdc.kugi.kyoto-u.ac.jp/dst_realtime/201301/index.html).

Fig. $4 \mathrm{~b}$ shows the information on the elevation angle and the VTEC value of the satellite PRN 20. A notable point is
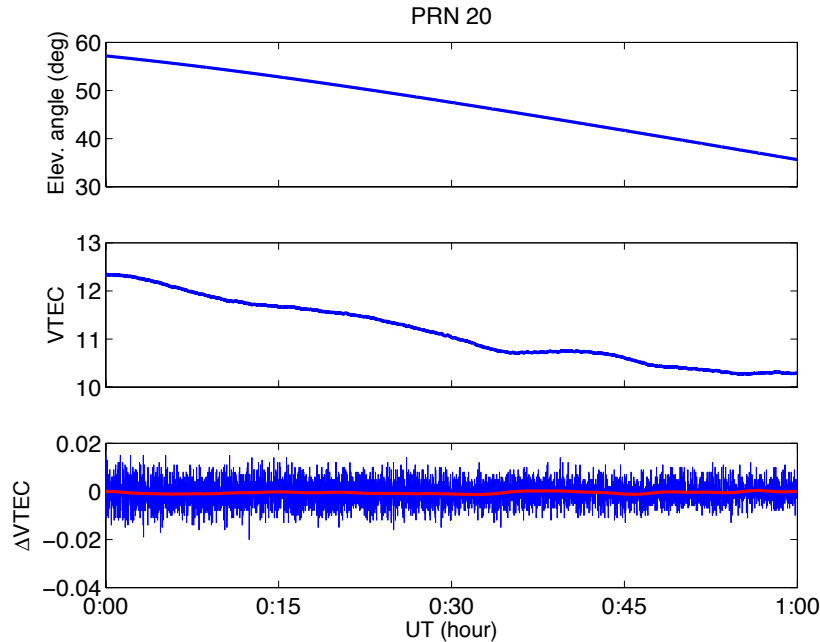

(b)
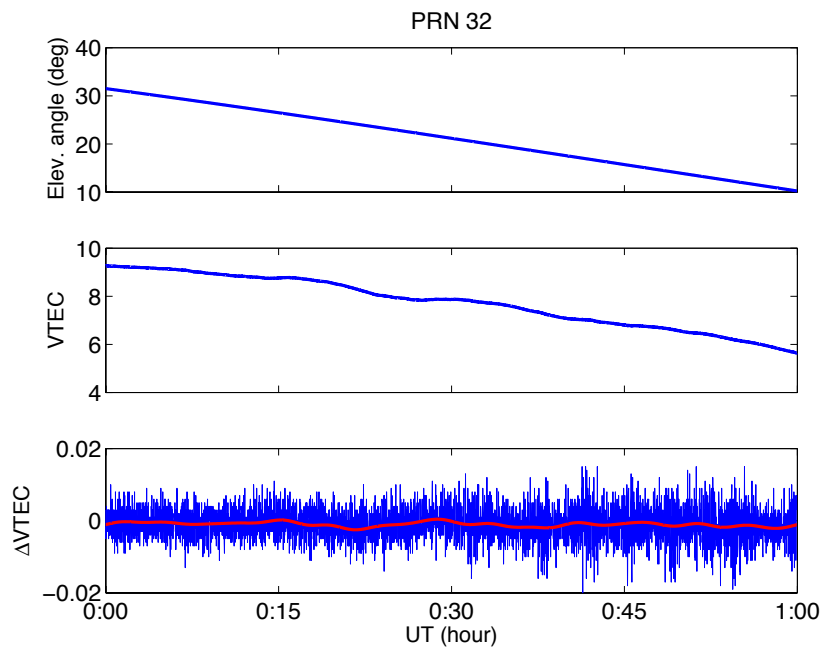

(d)

Fig. 4. Real-time ionospheric TEC values and variations. (a) PRN 4, (b) PRN 20, (c) PRN 23, (d) PRN 32 
that the variation range of the VTEC values depending on time decreased although the elevation angle of the satellite decreased. This is thought to be related with the signal-tonoise ratio of the GPS signals received at the GNSS receivers, but more detailed research (e.g., performance analysis of the receivers) is needed to find the cause. Fig. $4 \mathrm{c}$ shows the obtained information of the satellite PRN 23, which is similar to the result of the satellite PRN 4 in Fig. 4a. After the data processing, slight changes in the VTEC values depending on time were observed within 15 minutes. This is thought to be because the increased observation noise affected the variation of the VTEC values since the elevation angle of the satellite was less than 15 degrees.

Fig. $4 \mathrm{~d}$ shows the data processing result of the satellite PRN 32. The elevation angle of the satellite decreased from 32 degrees to 10 degrees during 1 hour, and the VTEC value also decreased by more than 3 TECU. For the variation of the VTEC values of the satellite PRN 32 depending on time, the variation range of $\triangle V T E C$ values increased as the elevation angle decreased.

When the elevation angle of a GPS satellite is low, it generally takes more time for the navigation signals transmitted from the satellite to pass the ionosphere, which increases the error or the observation noise. The results in Figs. $4 \mathrm{c}$ and $4 \mathrm{~d}$ support the above-mentioned explanation. However, the result in Figure $4 \mathrm{~b}$ is inconsistent with the above explanation, and there can be various causes for this inconsistency. It could be due to the characteristics of the GPS signal-to-noise ratio at a specific elevation, or the problem of the receiver hardware performance. Alternatively, it could be because the elevation angle of the satellite PRN 20 was maintained above 25 degrees during the data processing period, whereas the satellite PRN 23 and the satellite PRN 32 had a lower elevation angle. This needs to be addressed carefully since the characteristics could differ depending on GNSS receivers or satellite signals.

\section{CONCLUSION}

In this study, a real-time ionospheric TEC monitoring study was performed using the data from the nine GNSS reference stations operated by the Korea Astronomy and Space Science Institute. The data processing was carried out by converting the $1 \mathrm{~Hz}$ raw data, which is transmitted from each GNSS reference station, to RINEX every 5 minutes. The data processing period was 1 hour, and the elevation angle of satellites, the VTEC values, and the variation of the VTEC values were obtained for each GPS satellite PRN. The analysis indicated that the ionospheric VTEC values over the Korean peninsula did not show a large variation, and the variation of the VTEC values depending on time was also stable. However, for the satellite PRN 20, the variation of the VTEC values depending on elevation angles showed a trend that is different from other satellite signals. As this phenomenon is related with the observation noise of the GPS signals, further research is thought to be needed.

In this study, the research method for a real-time ionospheric TEC monitoring study was presented. Also, the VTEC values and the variation of the VTEC values depending on time were calculated and analyzed. These results are expected to be useful for the fields that utilize ionospheric TEC values in real time.

\section{ACKNOWLEDGEMENTS}

This study was supported by the 2013 Leading Core Technology Project funded by Korea Astronomy and Space Science Institute (KASI) [project name: Development of High Accuracy GNSS Data Analysis Engine]

\section{REFERENCES}

Afraimovich EL, Kosogorov EA, Lesyuta OS, Geomagnetic control of the spectrum of traveling ionospheric disturbances based on data from a global GPS network, Ann Geophys, 19, 7, 723-731 (2001)

Artru J, Ducic V, Kanamori H, Lognonne P, Marakami M, Ionospheric detection of gravity waves induced by tsunami, Geophys J Int, 160, 840-848 (2005).

Blewitt G, Hammond WC, Kreemer C, Plag HP, Stein S, et al., GPS for real-time earthquake source determination and tsunami warning systgems, J Geod, 83, 335-343 (2009). http://dx.doi.org/10.1007/s00190-008-0262-5

Calais E, Minster JB, GPS, earthquakes, the ionosphere, and the space shuttle, Phys. Earth Planet Inter, 105, 167-181 (1998).

Choi BK, Cho JH, Lee SJ, Estimation and analysis of GPS receiver differential code biases using KGN in Korean Peninsula, Adv Space Res, 47, 1590-1599 (2011).

Davis K, Hartmann GK, Studying the ionosphere with the Global Positioning System, Radio Sci, 32, 1695-1703 (1997).

Fedrizzi M, de Paula E, Kantor IJ, Langley R, Komjathy A, et al., Study of the March 31, 2001 magnetic storm effects on the ionospheric GPS data, Adv Space Res, 36, 534545 (2005).

Gao Y, Liu Z, Precise ionosphere modeling using regiona 
GPS network data, J Global Positioning Syst, 1, 18-24 (2002).

Heki K, Ionospheric electron enhancement preceding the 2011 Tohoku-Oki earthquake, GRL, 38, L17312 (2011). http://dx.doi.org/10.1029/2011GL047908

Kleusberg A, Kinematic Relative Positioning Using GPS Code and Carrier Beat Phase Observations, Marine Geod, 10, 257-274 (1986).

Klobuchar JA, Ionospheric Time-Delay Algorithm for SingleFrequency GPS User, IEEE Trans Aero Electro Sys, AES23, 325-331 (1987).

Komjathy A, Global Ionospheric Total Electron Content Mapping Using the Global Positioning System, PhD Thesis, University of New Brunswick (1997).

Komjathy A, Galvan DA, Stephens P, Butala MD, Akopian V, et al., Detecting ionospheric TEC perturbations caused by natural hazards using a global network of GPS receivers: The Tohoku case study, Earth Planets Space, 64, 1298-1294 (2012).

Mannucci A, Iijima B, Sparks L, Pi X, Wilson BD, et al., Assessment of global TEC mapping using a threedimensional electron density model, J Atmos Sol Terr Phys, 61, 1227-1236 (1999).

Mannucci A, Wilson BD, Yuan DN, Ho CH, Lindqwister UJ, et al., A global mapping technique for GPS-derived ionospheric total electron content measurements, Radio Sci, 33, 565-582 (1998).

Otsuka Y, Ogawa T, Saito A, Tsugawa T, A new technique for mapping of total electron content using GPS network in Japan, Earth Planets Space, 54, 63-70 (2002).

Prikryl P, Ghoddousi-Fard R, Kunduri B, Thomas E, Coster A, et al., GPS phase scintillation and proxy index at high latitudes during a moderate geomagnetic storm, Ann Geophys, 31, 805-816 (2013).

Sardon E, Rius A, Zarraoa N, Estimation of the transmitter and receiver differential biases and the ionospheric total electron content from Global Positioning System observations, Radio Sci, 29, 577-586 (1994).

Skone S, Wide area ionosphere grid modeling in the auroral region, $\mathrm{PhD}$ thesis, University of Calgary (1998).

Spogli L, Alfonsi L, Franceschi G, Romano V, Aquino M, et al., Climatology of GPS ionospheric scintillations over high and mid-latitude European regions, Ann Geophys, 27, 3429-3437 (2009) 